\title{
VISIÓN ESTRATÉGICA SOBRE LA GESTIÓN DE LA CALIDAD DE LAS INSTITUCIONES DE EDUCACIÓN SUPERIOR EN EL ECUADOR.
}

\section{STRATEGIC VISION ON QUALITY MANAGEMENT OF HIGHER EDUCATION INSTITUTIONS IN ECUADOR.}

\author{
Aida Isabel Jaya Escobar, Mgs. \\ Magíster en Administración de Empresas (Ecuador). \\ Docente de la Universidad Estatal de Bolívar (UTB), Ecuador. \\ jayaisabel@yahoo.es
}

Paola Estefanía Albán Trujillo, Mgs. Magíster en Gestión de Proyectos Socio Productivos (Ecuador). Docente de la Universidad Estatal de Bolívar (UTB), Ecuador. palban@ueb.edu.ec

Duniesky Alfonso Caveda, PhD. Doctor en Ciencias Pedagógicas (Cuba). Docente de la Facultad de Derecho y Gobernabilidad en la Universidad ECOTEC,

Ecuador. dalfonso@ecotec.edu.ec

ARTÍCULO DE REFLEXIÓN

Recibido: 2 de febrero de 2017.

Aceptado: 24 de abril de 2017.

\section{RESUMEN}

En este artículo se estudia la gestión de la calidad de las instituciones de educación superior en el Ecuador y sus retos a partir de los múltiples factores que determinan en la actualidad los niveles de eficiencia y pertinencia de las instituciones de educación superior (IES). De tal forma, aspectos como: la globalización, la competencia global, los cambios tecnológicos y las expectativas novedosas de los clientes, inciden en los parámetros 
reconocidos por los organismos rectores de la educación superior ecuatoriana para determinar la mayor o menor calidad de la formación profesional y su reconocimiento social como ventaja competitiva ante el resto de las universidades ecuatorianas.

Palabras clave: calidad educativa, enfoque estratégico, profesorado universitario.

\section{ABSTRACT}

In this article we study the quality management of higher education institutions in Ecuador from an approach caused by multiple factors such as: technological, globalization, global competition and the changing expectations of clients, pose new difficulties to the managers of the Organizations, through a strategic vision that contemplates the course of a quality management in higher education, considering that the quality of a service is essential to achieve a competitive advantage in Higher Education in Ecuador, despite all the evaluation Of the higher education service is complex not only because of the particular characteristics that this service must provide but because there are currently general evaluation parameters that must be met.

Keywords: educational quality, strategic approach, university teaching staff.

\section{INTRODUCCIÓN}

Los grandes cambios estructurales originados por múltiples factores: los cambios tecnológicos, la globalización, la competencia global y las expectativas cambiantes de los clientes, entre otros, plantean nuevas dificultades a los gestores de las organizaciones. Las antiguas recetas sobre las mejores formas de gestión de las organizaciones no funcionan ante los entornos más complejos y poco predecibles, por lo que los ejecutivos están dejando atrás los enfoques tradicionales e intentando nuevas fórmulas que fijen el rumbo en un entorno cambiante. En este sentido, adquiere particular importancia para las organizaciones la aplicación del enfoque estratégico, pues dota a la empresa de mayor flexibilidad, capacidad de adaptación, anticipación y actuación.

Cuando en la literatura se habla de "enfoque" se refiere a determinada orientación, concepción, visión o posición sobre algún fenómeno en cuestión. Mientras que la palabra estratégico encierra un conjunto de elementos como: visión, proyección, largo plazo y prospectiva. La segunda refuerza lo importante, necesario y trascendental de este enfoque para las organizaciones, ya que les permite a partir de una adecuada planificación, prevenir, anticiparse, actuar, adaptarse y romper barreras que favorecen el 
paso de un estado actual a un estado óptimo deseado (Mintzberg, Brian Quinn, \& Voyer, 1997)

En el Ecuador la gestión universitaria es tradicionalista. En algunas universidades se realizan procesos de planificación y evaluación institucional repetitivos y desvinculados entre sí, no hay acciones prospectivas que vayan en sentido de una interacción efectiva con la sociedad, puesto que no se evidencia una relación sistémica en sus procesos deteriorando de esta manera la concepción filosófica y el enfoque de calidad que debe existir en las instituciones educativas, el desempeño de una parte de sus directivos universitarios se orienta al comportamiento habitual y es inercial, tomándose decisiones de forma reactiva ante los problemas que se presentan.

Es así que en su actuación prima la estabilidad, con un grado mínimo de autorregulación; predomina el imperativo de evitar la crítica y el fracaso, y gestionan sin establecer prioridades, pues existe adicción a lo urgente, dejando a un lado lo importante, muchos de ellos, son prisioneros de lo que está pasando al interior de la universidad, y no se preocupan por el análisis del impacto de los resultados en el entorno, es decir, se encuentran atrapados y controlados por el funcionamiento interno, gustan de respuestas ya experimentadas, con un riesgo mínimo.

Por consiguiente, el temor al fracaso limita que apliquen nuevos enfoques e ignoren $u$ oculten los problemas institucionales, hasta que se hacen insoportables. Estas y otras insuficiencias, son barreras hoy presentes en la gestión universitaria en muchas instituciones ecuatorianas lo que impiden el cumplimiento de su objeto social y las desnaturalice de su verdadera esencia.

Es lamentable, pero estos viejos hábitos resisten con tenacidad extrema, ya que sus raíces son en esencia, un problema cultural, forman parte de los comportamientos y actitudes existentes en algunas comunidades universitarias. Esta manera de reflexionar y actuar, caracteriza el "estancamiento" y la "artritis organizacional" actual de algunas universidades ecuatorianas. Por ello se requiere romper esquemas y cambiar la cultura tradicionalista.

Dentro de las demandas que se le presenta a la Educación Superior, salta a la luz la problemática de la gestión de la calidad universitaria. En menos de una década, la preocupación por el mejoramiento constante de la calidad que antes era irrelevante, se ha transformado en una cuestión decisiva y crucial para cumplir con los retos señalados. Es 
por ello que ocupa actualmente un espacio mayor en la agenda de discusión de los organismos que dirigen la Educación Superior en Ecuador.

Frente a esta crítica situación, se hace necesario poner en marcha políticas y estrategias nacionales e institucionales para la educación superior con una visión estratégica, acompañadas de procesos de gestión y de evaluación de carácter innovador. Como modesta contribución a este empeño proponemos a través de este trabajo exponer como el enfoque estratégico influye en la gestión de la calidad en la educación superior, calidad de las IES en el Ecuador como los nuevos restos que debe asumir la dirección estratégica en la educación superior objetivo de la presente investigación.

\section{REVISIÓN TEÓRICA}

Fundamentación del enfoque estratégico en la gestión de la calidad en la educación superior.

El mundo actual es altamente competitivo, las tecnologías avanzan a un ritmo sorprendente y los clientes son cada vez más exigentes con respecto a productos o servicios con características que satisfagan sus necesidades y expectativas. Estos cambios que inciden en todos los órdenes de la vida de las colectividades, regiones, estados nacionales, y que generan riesgos, incertidumbre e inestabilidad, demandan de la universidad una gestión acorde con estas nuevas circunstancias.

Las organizaciones del siglo XXI deben estar dirigidas por sistemas planificados de gestión. Uno de los aspectos más importantes a considerar es la gestión de la calidad como una filosofía que impacta a todos los procesos de una institución: estratégicos, medulares y de apoyo.

Un sistema de gestión de la calidad según Peralta (2006), es el conjunto de normas interrelacionadas de una organización por los cuales se administra de forma ordenada la calidad de la misma, en la búsqueda de la mejora continua", por lo que la adopción de un sistema de gestión de calidad debe ser una decisión estratégica de la organización y este sistema está influenciado por el entorno de la organización, los cambios en ese entorno, las necesidades cambiantes, los productos que proporciona, los procesos que emplea, el tamaño y la estructura de la organización, el objetivo declarado en la Norma ISO 2008 es que la organización busque sin descanso la satisfacción del cliente a través del cumplimiento de los requisitos. 
Las Instituciones de Educación Superior (IES) y en especial las universidades, desempeñan un rol de suma importancia en la formación de recursos humanos del más alto nivel y en la creación, desarrollo, transferencia y adaptación de tecnología. De manera que lo que ellas hacen para responder adecuadamente a los requerimientos de la sociedad moderna, se constituya en un imperativo estratégico para el desarrollo nacional y se debe engranar con los planes de desarrollo del país. Dirigir y operar una organización en forma exitosa requiere ser transparente, flexible, sistemático, coherente y estar en relación constante y sistemática con el entorno. De la misma manera procurar que todos los procesos de la organización estén impregnados por la cultura de la mejora continua, de esta forma se puede lograr el éxito si se implementa y mantienen un sistema de gestión de la calidad.

De esta forma en estos últimos años, el pensamiento estratégico y la prospectiva constituyen herramientas invaluables para poder orientarse en los nuevos entornos, en contraposición a corrientes pesimistas que piensan que el futuro ya está dado, como un destino inexorable. Esta posición humana y proactiva recalca que podemos y debemos construir el futuro. Que tenemos que hacerlo como una necesidad y como una obligación, buscando siempre alcanzar los grandes sueños y utopías que las diferentes sociedades, organizaciones y personas, han hilvanado en la historia humana.

La planificación estratégica institucional debe ser coordinada con los niveles funcionales de la organización, como un proceso a través del cual se definen los objetivos de la universidad y las estrategias que posibilitan alcanzarlos. Se trata de un proceso de gobernar en la incertidumbre que debe ser comprendido como un instrumento de cambio. Para ello es importante tener en cuenta las políticas establecidas en el país en este sector y la misión institucional, las que constituyen el soporte del análisis estratégico que permitirá, a partir de la identificación de fuerzas internas, externas y su evaluación, proyectar opciones estratégicas para el período planificado. El plan propuesto debe ser eminentemente participativo y flexible, sin perder de vista una visión estratégica que se concrete en un pensamiento que sea síntesis de un pensamiento lógico, un pensamiento crítico y un pensamiento creativo.

En el modelo de gestión de la calidad para universidades del Ecuador, en particular en el caso de la Universidad Estatal de Bolívar se pretende identificar los ocho principios que establece la norma ISO IWA (2003) (Internacional Workshop Agreement) de aplicación en la educación, que se constituirán en las bases filosóficas para conducir la organización. La 
finalidad del modelo aplicado al ámbito educativo es conocer la realidad de la institución mediante la autoevaluación, evaluación y reflexionar sobre ella, lo que posibilitará elaborar planes y estrategias de mejora. Pretende analizar la organización de manera integral: la gestión del Proyecto Educativo Institucional desde el ámbito de la dirección, liderazgo, gobierno, pedagógico, administrativo, comunitario como su implementación y sus resultados (Hernández, 2009).

La propuesta de un Modelo de Calidad, supone la estructuración de un ciclo de aprendizaje en todos los procesos llevados a cabo en la institución. Para la instalación del Modelo de Gestión de la Calidad, se propone la utilización de una herramienta sistémica que permita alinear las iniciativas y esfuerzos individuales hacia el logro de los objetivos estratégicos, para demostrar a la comunidad universitaria que como institución se está creando valor para la sociedad; para los usuarios internos y externos; para la propia universidad y para el talento humano.

La sociedad actual se encuentra inmersa en la búsqueda de alternativas educativas que permitan pensar y actuar en el futuro conforme a los venideros cambios. Eso obliga a revisar con atención el modelo educativo vigente y sobre la base, proponer el mejoramiento de la calidad educativa y por tanto, replantear el papel que desempeñan las IES, lo que permitirá descubrir y crear nuevos vínculos entre la educación y la sociedad.

\section{La calidad en las instituciones de educación superior.}

Hasta hace algunas décadas, el término calidad educativa no era una prioridad en los planes de desarrollo educativo de los gobiernos e instituciones; era más importante la masificación, la cobertura, el financiamiento, la descentralización, etc., que la calidad de la educación como proyecto de mejoramiento personal y social de desarrollo nacional.

La búsqueda de la calidad tiene muchas facetas y va más allá de una interpretación estrecha del rol académico de los diferentes programas. Sobre la base de las consideraciones anteriores también implica prestar a cuestiones con referente a la calidad de los estudiantes la infraestructura, así como del entorno académico de todos estos aspectos se debe considerar que un estudiante debe tener variadas facetas como: académica, profesional, personal, social y administrativa unidos al interés por un buen gobierno, representan una manera importante en la determinación del funcionamiento de determinada institución, en la forma en que es evaluada y la imagen institucional, que 
puede proyectar hacia la comunidad académica como la sociedad en general (García, 2008).

En Ecuador luego de la llegada de la Revolución Ciudadana al Gobierno, una de las características distintivas que tomó el sistema de educación superior fue la evaluación de sus instituciones. En este contexto será factible, el modelo de gestión de la calidad de la Universidad Estatal de Bolívar propuesto por la autora como resultado de su investigación. El mismo deberá proyectarse con un enfoque integral, atendiendo a los ejes prioritarios concebidos para el desarrollo de las IES en Ecuador. El modelo que se pretende proponer será descriptivo, secuencial, dinámico, abierto, con aplicación de procesos, basado en los principios de la guía IWA2, enmarcadas en etapas como: la revisión de la dirección, gestión de recursos, realización del producto y medición, análisis y mejora, esto requiere un enfoque analítico sobre el marco legal que regula y estructura el tema en discusión, es entonces en donde se destaca el desarrollo secuencial de la estrategia como parte de una planeación anual en donde se determinan los factores internos y externos del entorno por lo que se despliegan los subsistemas como todos los instrumentos que permiten su realización hasta el desempeño de acuerdo con los postulados de la escuela de la Planificación.

\section{Importancia de la dirección estratégica en las instituciones de educación superior.}

Según la Declaración de la Conferencia Regional de Educación Superior (CRES), esta tarea es decisiva para las IES en la actualidad, pues exige formular nuevas acciones centradas en los puntos claves siguientes:

- La participación de la comunidad universitaria en el proceso de toma de decisiones.

- El desarrollo de sistemas alternativos de evaluación y acreditación más pertinentes.

- La profesionalización de los directivos.

- El fortalecimiento de la vinculación entre la misión y los sistemas de gestión (IESALC, 2008).

A lo anterior se puede añadir que el mejoramiento de la calidad de la gestión universitaria demanda además de otros elementos esenciales: aprendizaje organizacional, rapidez, innovación, flexibilidad y capacidad institucional para adaptarse a los requerimientos del entorno y transformarlo para contribuir al desarrollo sostenible de los países. 
Para lograr el cambio se hace necesario desaprender los viejos conocimientos organizacionales y los comportamientos del personal directivo lo que exige la creación y divulgación de nuevos conocimientos a través de procesos de capacitación, estudios e investigaciones en el ámbito de la gestión universitaria; el desarrollo de una actitud emprendedora de los directivos, sin temer al cambio; el reconocimiento de los errores actuales en la gestión; la comparación como el análisis de las buenas prácticas; el trabajo en equipo, y la colaboración tanto interna como externa.

Superar los paradigmas actuales de la gestión universitaria tradicional, no es una tarea sencilla, por ello no en todas las IES se ha logrado. Como sucede con cualquier cambio drástico de paradigmas, el proceso de construir lo nuevo no es instantáneo, al contrario, a veces es lento lo que conlleva la alta resistencia, lo cierto es, que los nuevos enfoques que van apareciendo, se contraponen a algunos rasgos de la gestión universitaria tradicional, que ya se encuentran desactualizados y han sido superados.

$\mathrm{Si}$ los elementos claves señalados logran convertirse en componentes integrales de la gestión universitaria, entonces será posible avanzar significativamente en la calidad y pertinencia de sus resultados. Es decir, se transforman en la protección contra la enfermedad y debilitación institucional por tanto pasan a formar parte de la nueva visión que exige una gestión universitaria más competente.

La aplicación del enfoque de dirección estratégica se convierte actualmente en una necesidad de las IES, sobre todo en las públicas, ya que las mismas son organizaciones complejas que se mueven en múltiples esferas académicas. A esta disyuntiva debe dar respuesta el enfoque estratégico orientado a la promoción de la calidad y la excelencia como factores claves de éxito. Ello requiere abrir procesos de reflexión sobre la naturaleza de las acciones, así como incluir orientaciones a mediano y largo plazo en la agenda de las IES. La dirección estratégica significa concebir a la IES mirando siempre hacia un futuro factible a lograr, donde muchas de las acciones repetitivas y homogéneas están descontextualizadas (Almuiñas, 1999).

De los anteriores planteamientos se deduce que la dirección estratégica es un proceso que afecta a todos los niveles de decisión y funciones en una IES. Requiere del diseño de herramientas que guíen la manera de lograr los objetivos estratégicos formulados. Sus paradigmas claves son: el pensamiento estratégico, la actitud estratégica la misión, los valores, la visión, los objetivos, las estrategias específicas y su implementación, seguimiento y evaluación (Ronda, 2007). 
Para las IES, concebir la dirección estratégica exige enfrentar un conjunto de desafíos como: establecer fuertes relaciones con el entorno, tanto del macro-entorno como del micro-entorno, cambiar la cultura organizacional en función de las demandas, alinear la estrategia institucional con las políticas públicas de la Educación Superior; concentrar los esfuerzos en las prioridades institucionales definidas; elevar el nivel de participación e implicación de la comunidad universitaria en el proceso de toma de decisiones estratégicas; desarrollar un pensamiento como una acción emprendedora; lograr una adecuada integración estructural y funcional con los restantes procesos universitarios; alinear los sistemas de evaluación institucional, control estratégico como otros, que contengan acciones evaluativas; comunicar adecuadamente la estrategia en la organización, tanto vertical como horizontal así como también interna y externa, finalmente desarrollar el liderazgo colectivo y el aprendizaje organizacional para favorecer un desempeño organizacional eficiente y eficaz.

Lo señalado debe ser parte del desarrollo de los soportes estratégicos y en consecuencia deben ser tomados en cuenta como elementos imprescindibles para la puesta en marcha de las estrategias.

\section{Retos de la dirección estratégica en las instituciones de educación superior.}

Teniendo en cuenta las aportaciones de Bustos et al. (2008), referidas a la dependencia del direccionamiento estratégico en relación con la dirección de las organizaciones, para el éxito o fracaso en las mismas, revela la coherencia que existe entre los indicadores de Jerarquía como de la planificación estratégica de las IES, lo que conlleva a que la dirección estratégica se enfoque a fortalecer los procesos de autoevaluación para el aseguramiento de la calidad y por ende la mejora de su pertinencia académica y social.

Estos procesos deben tener carácter permanente y facilitar el tránsito de una"cultura de la evaluación" a una "cultura de planificación y gestión estratégica responsable, autónoma, pertinente y eficiente" en cada institución. Para ello, debe procurarse contar con estructuras institucionales de carácter innovador, con nuevas modalidades de planificación, de conducción, de evaluación y de toma de decisiones, que tengan carácter democrático y participativo, como ya ha sido planteado. Dentro de ese punto de vista, debería tenerse en cuenta que el desarrollo institucional a mediano y largo plazo se sustente en planes y programas de carácter estratégico, articulados con los requerimientos nacionales y de la región en que esté instalada la institución, y 
establecidos con una orientación prospectiva. Para ello, las estructuras organizativas deberán ser flexibles e innovadoras, y basarse en una reingeniería en profundidad de los procesos de gestión.

En este marco, el funcionamiento de las instituciones universitarias requiere ser articulado desde su autonomía con los sectores del trabajo y la producción, con las organizaciones sociales y con todos los ámbitos públicos y privados pertinentes. En cuanto a la calidad debería asumirse una concepción institucional, basada en una "construcción social", de carácter gradual y colectivo, pertinente, sustentada en un proceso de reflexión y debate intra y extrauniversitario. En este caso, las instituciones universitarias deberían funcionar en un ámbito de creatividad, de innovación, de mirada hacia el futuro. Deberían como ya lo hemos señalado planificar estratégicamente, ejecutar con responsabilidad, hacer seguimiento y control, y evaluar, como proceso permanente y autorregulado. Esto facilitará la flexibilización y profesionalización de las estructuras de gestión institucional, académica y administrativa, con sentido integrador, y utilizando las tecnologías más eficientes.

La universidad debe trabajar efectivamente para alcanzar consensos básicos en materia de políticas públicas a través de procesos de concertación, para promover nuevas modalidades de representación social, para establecer canales institucionales a las demandas de participación social, para el desarrollo y aceptación colectiva de valores ético-morales, en el marco de una cultura cívica democrática para la consolidación en sus estudiantes y graduados, de actitudes y juicios críticos sobre instituciones, procesos y actores, y para que la educación y la universidad se constituyan nuevamente en medios eficientes de movilidad e integración social.

Es así que la gobernabilidad democrática de los sistemas educativos y de las instituciones universitarias requiere asumir a la educación como una tarea de todos, con la centralidad del Estado, con una actitud pluralista y negociadora, con decisiones adoptadas en forma participativa consensuada en el contexto de una nueva relación con el Estado y la sociedad.

Almuiñas Rivero \& Galarza Lopez ( 2016), proponen un modelo teórico-funcional sustentado en procesos para la planificación estratégica en las IES la generación continua de nuevos conocimientos y la formación integral de las personas, con valores que respondan a las necesidades de la sociedad; una democratización del acceso, permanencia y egreso; una mayor articulación de la Universidad con los niveles 
educativos precedentes; el desarrollo de la investigación científica y el postgrado; el mejoramiento de la extensión; una mayor aplicación de las TIC, como apoyo a los diferentes procesos universitarios; el fortalecimiento de la cooperación internacional; la mayor participación de la comunidad universitaria en la toma de decisiones institucionales; el desarrollo de sistemas adecuados de evaluación, y la formación específica de los directivos para poder gestionar adecuadamente estos procesos.

Es necesario diseñar y ejecutar planes estratégicos tanto para el conjunto del sistema universitario como para cada institución en un marco amplio y participativo que tienda a la búsqueda de consensos, o la discusión ordenada de disensos entre todos los actores pertinentes intra y extrauniversitario, esto requiere articularse fuertemente con la sociedad y sus organizaciones representativas, tendiendo a establecer una nueva alianza sociedaduniversidad, que posibilite asumir sus demandas y facilite su participación efectiva en el diseño y desarrollo de las políticas sectoriales e institucionales.

Los planes estratégicos universitarios deben articularse con las políticas y planes del conjunto de la educación de manera de asegurar su integración y con las del sistema científico-tecnológico, para responder con pertinencia a sus desafíos. Asimismo, deben coordinarse con los sectores del trabajo y de la producción, de manera de contribuir a su mejoramiento tecnológico y a su mejor funcionamiento en el marco de los requerimientos globales, nacionales y regionales.

Estas vinculaciones requieren de una educación superior diseñada para su desarrollo a lo largo de toda la vida. La evaluación institucional analiza estas dimensiones, por lo tanto, sería importante se señala que se tome como un proceso necesario y útil para la revisión de la planificación y gestión estratégica de las Instituciones de Educación Superior.

\section{MATERIALES Y MÉTODOS}

La investigación se sitúa como un paradigma critico - propositivo, según Chicaiza (2015), es crítico porque considera la investigación como una interpretación, comprensión y explicación de los fenómenos sociales que influyen en la gestión de la calidad de servicio en la educación superior en las Universidades del Ecuador. Propositiva por cuanto plantea una alterativa de solución al problema investigado, partiendo de la esencia que tiene los fenómenos sociales a partir de las interacciones y contradicciones que genera los cambios cualitativos en una investigación. 
La investigación es crítica, debido a que al conocer la importancia de la dirección estratégica en las instituciones de Educación Superior en el Ecuador exige formular nuevas acciones concentradas en la excelencia, basados en competencias en la educación superior, lineamientos metodológicos y profesionalización de los directivos. En este contexto la gestión de las Universidades del Ecuador sigue siendo tradicionalista. Al percibir la visión estratégica en las Instituciones de Educación Superior del Ecuador, se puede implementar nuevos modelos de gestión estratégica que mejore la calidad de Educación Superior en el Ecuador.

La representación científica de la presente indagación se evidencia en fundamentos epistemológicos que sustentan un modelo de investigación cualitativa en las ciencias sociales (Parra \& Recasens Salvo, 2005).

De todo lo anterior señalado la presente investigación se basa en un enfoque empírico, análitico a partir de la explicación de la importancia que tiene la gestión en lo que respecta en la calidad de Educaión Superior de las Instituciones del Ecuador, concluyendo en un enfoque fenomeológico-hermenéutico que permite comprender e interpretar la visión estrategica que tienden sobre la gestión que llevan los directivos de las Universidades del Ecuador.

\section{CONCLUSIONES}

La necesidad de implementar nuevos modelos de gestión dinámicos, abiertos, normativos con un sistema categorial definido que combine la calidad de los procesos con los factores externos teniendo como enfoque la calidad de la educación superior.

Un enfoque estratégico no solo es aplicable en el contexto organizacional, su esencia y concepción puede servir de referente para las investigaciones científicas, a partir de reconocer y utilizar el sistema categorial con que trabaja, el cual le impregna una visión o perspectiva de análisis diferente.

El transitar de la "cultura de la evaluación" a la "cultura de una gestión autónoma, pertinente, responsable y eficiente" con una nueva concepción estratégica sobre planificación y gestión de la educación superior, en la que la evaluación, la acreditación y el aseguramiento de la calidad sean procesos permanentes a través de fortalecer los estudios y debates a nivel nacional y regional con carácter colectivo y participativo y comparado, organizando redes, asociando esfuerzos de agencias nacionales y 
regionales, organismos de cooperación técnica, de organismos internacionales y regionales.

\section{REFERENCIAS BIBLIOGRÁFICAS}

Peralta, Y. (2006). Mitos y realidad des ISO 9001:2000 en organizaciones educativas. Mexico: Limusa.

Almuiñas Rivero, J. L. (1999). La planificación estrátegica en las Instituciones de Educaión Superior. Cuba: Universidad de la Habana.

Almuiñas Rivero, J. L., \& Galarza Lopez , J. (2016). Las redes académicas como ejes de integración y cooperación internacional de las instituciones de educación superior. Revista Cubana de Educación Superior, 18-29.

Bustos, J., Zapata, M., \& Valdivia, M. T. (2008). Más allá de la gestión estratégica en educación superior:aplicación del Cuadro de Mando Integral. Oikos:Revista de la Escuela de Administración y Economía, 95-114.

Chicaiza , M. (25 de Marzo de 2015). prezi .com. Obtenido de https://prezi.com/6mu3d8gdjevw/paradigma-critico-propositivo/

García Nieto, N. (2008). La función tutorial de la Universidad en el actual contexto de la Educación Superior. Interuniversitaria de Formación del Profesorado, 21-48.

Hernández, B. I. (2009). Dieño de un modelo de gestón integral para las Instituciones de Educaión Superior, basado en los lineamientos para la autoevaluación con fines para la acreditación del Consejo Nacional de Acreditación (CNA). Cartagena : Universidad de Cartagena. 
IESALC, U. (2008). Declaración de la Conferencia Regional sobre Educación para América Latina y el Caribe. En Conferencia Regional sobre educaión para América Latina y el Caribe. Cartagena de Indias.

Mintzberg, H., Brian Quinn, J., \& Voyer, J. (1997). El Proceso Estratégico,conceptos,contextos y casos. México: Pearson Prentice Hall.

Parra Sabaj, M. E., \& Recasens Salvo, A. (2005). Fundamentos Epistemológicos, Metodológicos y teóricos que sustentan un modelo de investigación cualitativa en las ciencias sociales. Chile.

Ronda , G. (2007). Se refiere a la actitud anticipadora, crítica,extrivertida y aierta al cambio.

\section{Licencia Creative Commons}

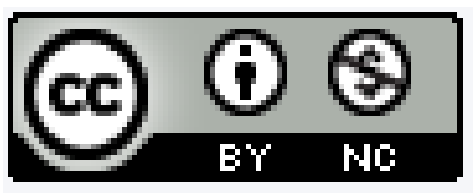

Revista Científica ECOCIENCIA está bajo una Licencia Creative Commons Atribución-NoComercial 4.0 Internacional. 